\title{
Marktzugangsregulierung: Nationale Regulierung, europäische Integration und internationale Harmonisierung in der Arzneimittelzulassung ${ }^{1}$
}

Jürgen Feick

In diesem Beitrag geht es um die Untersuchung der Wechselbeziehungen zwischen den Bedingungen von Marktentfaltung und politischer Intervention am Beispiel der Regulierung der Marktzulassung von Fertigarzneimitteln ${ }^{2}$. Auf nationaler Ebene wird die Entwicklung in den Ländern Deutschland, Frankreich und England untersucht, darüber hinaus die auf europäischer Ebene sowie erste Schritte internationaler Harmonisierung zwischen den großen Regionen Nordamerika (USA), Europa und Asien (Japan). Bei aller gebotenen Kürze der empirischen Darstellung sollen die Reaktionen der nationalen bzw. transnationalen Regelungssysteme auf spezifische Problem- und Interessenlagen und speziell die Bedingungen und Grenzen einer sich sukzessive durchsetzenden regulativen Supranationalisierung und beginnenden internationalen Harmonisierung analysiert werden. Schließlich ist danach zu fragen, welche Auswirkungen die jeweiligen regulativen Reaktionen auf konkrete Marktkonstellationen haben.

Von Arzneimittelmärkten zu sprechen, heißt Tauschbeziehungen als Märkte zu bezeichnen, die in vielfältiger Weise durch kollektive Interventionen beeinflusst oder sogar dominiert sind. Wenn Arzneimittel häufig als das am stärksten regulierte Produkt bezeichnet werden, dann ist hiermit im Wesentlichen die Spannbreite der Interventionen gemeint, die von der Forschungs- und Entwicklungsphase bis hin zur Verabreichung von Medikamenten reichen.

Abbildung 1 zeigt, dass jeweilige Teilregelungen auf vor- und nachgelagerte Phasen ausstrahlen können; so erweisen sich häufig Folgeregelungen als notwendig, die entweder die Ausgangsregelung zunehmend operationalisieren und präzisieren oder auch in andere Teilbereiche zusätzlich regulierend eingreifen. Beispielsweise wird zunehmend präzisiert, welche Merkmale die Qualität eines Arzneimittels definieren sollen, gleichzeitig muss der zukünftige Produktionsprozess kontrolliert werden, um die Einhaltung der Qualität zu garantieren. Umgekehrt sind die Kriterien der Sicherheit und Wirksamkeit von den Zulassungsbehörden nur dann valide zu evalu-

1 Susanne Lütz danke ich herzlich für inhaltliche Verbesserungsvorschläge sowie Marlies Kafczik und Philipp Botzenhardt insbesondere für Datenrecherchen und das Erstellen von Abbildungen.

2 Fertigarzneimittel oder Arzneispezialitäten sind Medikamente, die auf Vorrat für einen über den Lokalbereich hinausreichenden Markt in größeren Mengen hergestellt werden. Das Pendant zur Marktzulassung ist die Überwachung vermarkteter Arzneimittel. 




ieren, wenn durch Vorgaben sichergestellt wird, dass die vorklinischen und klinischen Prüfverfahren aussagekräftige Ergebnisse liefern.

Regelungen werden u.a. durch die Interventionsinstrumente charakterisiert, mit denen das Verhalten von Akteuren beeinflusst werden soll. ${ }^{3}$ In der Arzneimittelzulassung stehen die klassischen regulativen Interventionsinstrumente im Vordergrund: Gebote, Verbote bzw. bedingte Erlaubnisse. Sie können aber ergänzt oder flankiert werden von Instrumenten der prozeduralen Regelung von Selbststeuerungssystemen, finanziellen Anreizen oder auch Informations- und Beratungsangeboten bzw. Appellen. Dies spielt insbesondere bei den erwähnten Folgeregelungen für den Implementationsprozess eine Rolle, da das Arzneimittelzulassungsrecht in hohem Maße durch unbestimmte Rechtsbegriffe gekennzeichnet ist, welche erst im Prozess der Rechtsanwendung interpretiert und inhaltlich gefüllt werden müssen (vgl. D. Hart/N. Reich 1990).

\section{Gründe für die Regulierung des Arzneimittelmarktes}

Die moderne produktorientierte Zulassungsregulierung für Arzneimittel hat zwar Wurzeln, die bis zur Jahrhundertwende zurückreichen, in ihrer heutigen Form ist sie jedoch ein Geschöpf der Nachkriegszeit, nicht zuletzt veranlasst durch die die

3 Verschiedene Ansätze der Typologisierung von politischen Interventionen finden sich bei K. König / N. Dose (1993). 
Öffentlichkeit bewegenden Arzneimittelkatastrophen und beeinflusst durch die sich im Gefolge von Umwelt- und Verbraucherschutzbewegungen in den USA seit den Sechzigerjahren durchsetzenden „new social regulation“. Bei ihr steht die Kontrolle von Produkt-, Prozess- und Verhaltensrisiken für Mensch und Umwelt im Vordergrund. Diese neuen Regulierungen übten einen großen Einfluss auf entsprechende staatliche Interventionsprogramme in den europäischen Staaten aus. ${ }^{4}$

Bei der hier interessierenden Arzneimittelzulassung geht es zunächst einmal um den Schutz von Verbrauchern/Patienten vor unsicheren oder unwirksamen Produkten. Ein amerikanischer Arzt merkte bereits im vergangenen Jahrhundert während einer Versammlung der Massachusetts Medical Society an: „I firmly believe that if the whole materia medica, as now used, could be sunk to the bottom of the sea, it would be better for mankind and all the worse for the fishes" (M. Silverman/P. R. Lee 1974: 258, zit. nach J. Abraham 1995: 42). Damit reagierte er auch auf einen Arzneimittelmarkt in den USA, der nach dem Bürgerkrieg geprägt war von zunehmend aggressiver Werbung für Arzneimittel mit häufig nicht deklariertem (,„patented medicines“ oder Geheimmittel) oder auch gefälschtem Inhalt, die ein erhebliches Gesundheitsrisiko darstellen konnten. Das Gefährdungspotenzial wuchs mit der Industrialisierung der Produktion, die zunehmend die individuelle Herstellung nach Rezept in der Apotheke ablöste. Hinter dem Ausruf des amerikanischen Arztes verbirgt sich gleichzeitig die zunehmende Ratlosigkeit der ärztlichen Profession angesichts eines Marktes, der auch für den Arzt nicht mehr überschaubar ist. Die nur allmählich und zum Teil gegen erheblichen Widerstand der Industrie eingeführte und im Laufe der Jahre verschärfte Risikoregulierung im Arzneimittelbereich ${ }^{5}$ diente jedoch nicht nur der Funktion der Produktsicherheit, sie stellte auch einen Schutz für seriöse Arzneimittelhersteller gegenüber unseriöser Konkurrenz dar und versah die Produkte der exportorientierten Industrie mit einem gewissen Gütesiegel.

Aus diesen exemplarischen Hinweisen lassen sich systematisch folgende Gründe für die Zulassungsregulierung ableiten, welche im Wesentlichen auf Konzepten einer Theorie des Marktversagens bzw. der Marktunvollkommenheiten aufbauen. ${ }^{6}$ Mit dem Produkt Arzneimittel sind nicht nur positive Wirkungen, sondern in der Regel auch unerwünschte Nebenwirkungen verbunden, negative Externalitäten, deren direktes Risiko - in Abwesenheit entsprechender kollektiver Regelungen - der Konsument/Patient zu tragen hat. Dies könnte dann als akzeptabel betrachtet werden, wenn dieser zur Abschätzung solcher Risiken in der Lage wäre. Hier fehlt aber in aller Regel die notwendige Transparenz - und zwar nicht nur beim Patienten, sondern in hohem Maße sogar auf Seiten der behandelnden Ärzte. Diese fehlende

4 Einen Überblick über die US-amerikanische Diskussion Ende der siebziger Jahre unter wirtschaftswissenschaftlichem Blickwinkel und mit gleichzeitigem Bezug auf die deutschen Verhältnisse bieten J. Müller/I. Vogelsang (1979); vgl. J. Feick (1980) zur Kritik regulativer Politik in den USA.

5 Exemplarisch für die USA und Großbritannien, J. Abraham (1995: Kap. 2).

6 Eberlein geht in seinem Beitrag in diesem Band auf diese Diskussion ein. Zu den frühesten systematischen Beiträgen zur Theorie des Marktversagens, vgl. F. Bator (1958). 
Transparenz kann einerseits einem grundsätzlichen Mangel an Informationen geschuldet sein, der dadurch entsteht, dass prinzipiell mögliche Analysen, Tests und Kontrollen noch nicht durchgeführt worden sind. Zum anderen beruht ein möglicher Mangel an Transparenz aber auch auf der Informationsasymmetrie, die zwischen dem Hersteller einerseits und dem Arzt bzw. Patienten andererseits existiert. Die potenziell negativen Externalitäten werden darüber hinaus zu einem kollektiven Problem für die Volkswirtschaft oder die Gemeinschaft, wenn Patienten durch die Einnahme unsicherer oder unwirksamer Arzneimittel ihre Arbeitskraft verlieren oder ihre Krankheitskosten von kollektiv organisierten sozialen Sicherungssystemen mitgetragen werden müssen.

Damit sind Gründe genannt, die bei Arzneimitteln prinzipiell dafür sprechen, den Zugang zum Markt nicht einem Laissez-faire zu überlassen, sondern kollektiv wirksamen Regelungen zu unterwerfen. Ob solche Regelungen tatsächlich eingeführt werden, in welcher institutionellen Form und welcher inhaltlicher Ausprägung hängt allerdings nicht von den theoretisch ableitbaren Gründen ab; entscheidend ist vielmehr die konkrete Wahrnehmung von Mängeln und einem daraus hervorgehenden Problemdruck sowie Regelungsbedarf, der im Prozess der politischen Willensbildung und Entscheidungsdurchsetzung zu den jeweils spezifischen Regelungen oder auch ,Nicht-Entscheidungen` führt.

\section{3}

\section{Zur Situation der Arzneimittelindustrie: Vom „golden age“ zum regulativen Kosten- und internationalen Wettbewerbsdruck}

Nach dem Zweiten Weltkrieg beginnt das (erste) „golden age“ der Pharmaindustrie auf der Basis chemisch-synthetischer Arzneimittelentwicklungen, die neue kurative, zumindest aber symptomatische Behandlungen, ermöglichen. In den Ländern mit einer nennenswerten Arzneimittelproduktion gewinnt die Pharmaindustrie den Status einer Innovations- und Wachstumsindustrie, deren gesundheitspolitische und volkswirtschaftliche Bedeutung zunächst unbestritten ist. Es sind in der Folgezeit vor allem die Konsequenzen der großen Arzneimittelkatastrophe der Sechzigerjahre (Thalidomid/Contergan), das Abklingen der Innovationsdynamik in den Siebzigerjahren und schließlich die zunehmende Kritik an den die öffentlichen Gesundheitsbudgets belastenden Arzneimittelkosten, die diesen Glanz verblassen lassen. Arzneimittelregulierung im Sinne eines strikteren Verbraucher- und Patientenschutzes kommt in praktisch allen entwickelten Staaten ebenso auf die politische Agenda wie Kostendämpfungsmaßnahmen innerhalb öffentlicher oder kollektiver Gesundheitssysteme. Einen erneuten, zumindest partiellen Perspektiven- und Imagewechsel läuten die volkswirtschaftlichen Wachstumsprobleme ein, die in den entwickelten Industriestaaten spätestens seit Mitte der Achtzigerjahre die öffentliche Diskussion bestimmen, parallel dazu die neuen biotechnischen Perspektiven der Arzneimittelentwicklung. Damit erhalten die Innovations-, Wachstums- und insbesondere auch Arbeitsmarktimpulse einer florierenden und zur Aussenhandelsbilanz positiv beitragenden Pharmaindustrie wieder stärkeres Augenmerk. Entsprechend gewinnen auch 
schon vorher geäußerte Kritiken an regulativen Interventionen in den Arzneimittelmarkt deutlichere Konturen. Zwar stellt sich nicht wieder die fast blind-optimistische Orientierung der Nachkriegsjahre ein, und es wird nach wie vor ein kritisches Augenmerk auf die Sicherheitsaspekte und die Kostenbelastung durch den Arzneimittelkonsum geworfen, gleichzeitig jedoch auf Seiten der Industrie und Politik nach den Kosten der verschiedenen regulativen Interventionen gefragt.

In den letzten zwei Jahrzehnten erfährt die Pharmaindustrie einen Wandel in fast all ihren Aktivitäten, der - vielleicht übertreibend, aber doch charakteristisch für die Eigenwahrnehmung der Industrie und industrienaher Beobachter - als „monumental change“ charakterisiert wird. Was noch Anfang der Siebzigerjahre durch eine „atmosphere as an elite gentlemen’s club“ geprägt gewesen sein soll, habe sich in eine hochwettbewerbliche, in der Regel kosteneffizient organisierte und vor allem auf Forschungs- und Entwicklungserfolg ausgerichtete Industrie verwandelt (R. M. Nordmann 1997: 28-29). Dieser Wandel innerhalb der Industrie ist geprägt von folgenden Teilaspekten:

- nach einer Phase der Innovationsstagnation seit den Siebzigerjahren ist durch die Biotechnologie und die Genforschung eine neue Dynamik in die Medikamentenentwicklung gekommen;

- die Zunahme der Entwicklungskosten für innovative Medikamente, ${ }^{7}$ höhere Anforderungen an die Marktzulassung, die steigende Internationalisierung der Märkte, eine Politik der Kostendämpfung in praktisch allen öffentlichen Gesundheitssystemen und der Rationalisierung in so genannten Managed Care-Systemen verschiedener Ausprägung hat zu einer Verschärfung der Wettbewerbssituation und einer zunehmenden Differenzierung innerhalb der Pharmaindustrie geführt, deren Einzelinteressen auf Verbandsebene immer schwerer zu bündeln sind.

Den neuen Bedingungen begegnet ein Teil der Pharmaindustrie durch horizontale und vertikale strategische Aufkäufe, Zusammenschlüsse und Allianzen, mit deren Hilfe „economies of scale“ und „economies of scope“ erreicht werden sollen, die eine sich bislang insbesondere in den USA abzeichnende „diversification into health care services“ einschließen. Diese Strategie, bei der die Großen der Branche im Vordergrund stehen, erlaubt es, an verschiedenen strategischen Stellen der „value chain“" strategisch platziert zu sein (M. S. Roth 1997). Innerhalb der Pharmaindustrie hat seit Ende der Achtzigerjahre ein regelrechtes Wettrennen unter den großen Unternehmen um Aufkäufe, Fusionen und Allianzen eingesetzt, das immer neue Verschiebungen in den jährlichen Umsatzranglisten zur Folge hat. Diese Expansionsstrategie dient dazu, in Verteilungsnetzen international vertreten zu sein, ausreichendes Kapital für sich verteuernde Forschungs- und Entwicklungsaktivitäten zur Verfügung zu haben und die eigenen Forschungsaktivitäten oder Produktli-

7 Die heute zu veranschlagenden Forschungs- und Entwicklungskosten werden mit 200 bis 800 Mio. US-Dollar angegeben; vgl. Zahlen der US Biotechnology Industry Association in D. Green (1998a: II). 
nien zu ergänzen, um in beständiger Folge neue, noch patentgeschützte und höhere Preise ermöglichende Produkte auf den Markt zu bringen. Trotz gelegentlicher Kritik an dieser Gigantonomie wird von Unternehmensberatern und betreuenden Investitionsbanken in nächster Zukunft kein Ende dieser Entwicklung erwartet. Nur wenige Unternehmen mit ausreichend zukunftsträchtiger Produktpalette und finanziellem Rückhalt scheinen sich diesem Konzentrationssog entziehen zu können. ${ }^{8}$

Diese Entwicklungen haben die Differenzierung innerhalb der Industrie verstärkt, in der die großen, forschungsintensiven und kapitalstarken Pharmaunternehmen den innovativen Markt dominieren, während kleinere und mittlere Unternehmen in aller Regel auf Nischenmärkte, Nachahmerprodukte oder den stärker national orientierten Nichtverschreibungsmarkt ausweichen bzw. mit den großen kooperieren müssen. Aus dieser Differenzierung erwachsen unterschiedliche Interessenlagen gegenüber staatlicher Regulierung. Die innovationsstarke, in der Regel große und international orientierte Industrie kann sich sehr viel leichter auf strenge Regelungsanforderungen in der Zulassung einstellen als kleine und mittlere Unternehmen, und sie ist auch eher in der Lage nationale Regelungsvarianzen zu berücksichtigen, weil sie international orientiert und durch Niederlassungen oder Kooperationspartner in ausländischen Märkten wirkungsvoll vertreten ist. Diese Ausgangsannahmen lassen darauf schließen, dass die kleinen und mittleren Unternehmen am Offenhalten nationaler Märkte über gewohnte nationale Verfahren interessiert sind, während die von ihren Produkten her international wettbewerbsfähige Großindustrie einheitliche Marktzugangsbedingungen über Ländergrenzen hinweg bevorzugt.

Die Inter- bzw. Multinationalisierung der Pharmaindustrie ist nicht zuletzt der Ausweitung des internationalen Handels geschuldet. Dieser hat zwar eine lange Tradition, gewinnt aber nach einer Phase des (auch) regulativen Protektionismus erst mit der zunehmenden Liberalisierung des Welthandels nach dem Zweiten Weltkrieg und der Einrichtung von Freihandelszonen an Dynamik. Mittlerweile besteht eine hochgradige Verflechtung der drei großen nationalen/regionalen Pharmamärkte, die 1996 zusammen etwa 90\% des Weltpharmamarktes auf sich vereinten: die USA (31,7\%), Europa (EU + EFTA: 27,5\%) und Japan (20,8\%) (vgl. P. Evers 1998: 6). Diese Verflechtung spiegelt sich u.a. am Handelsaustausch selbst, an den jeweiligen gegenseitigen Marktanteilen und auch der internationalen Verteilung pharmazeutischer Großunternehmen wider, wobei allerdings die drei Regionen durchaus signifikant variieren. Vergleicht man die Anteile am Pharmamarkt verschiedener Regionen, zeigt sich, dass sich der Anteil amerikanischer Pharmaprodukte auf dem europäischen Markt (nur EU-Länder) innerhalb von zehn Jahren auf knapp 22\% mehr als verdoppelt hat; etwa gleich hoch ist der relativ konstante Anteil europäischer Produkte auf dem amerikanischen Markt. Der japanische Anteil an den beiden anderen regionalen Märkten weist zwar hohe Wachstumsraten auf, ist aber insgesamt noch verschwindend gering (unter 1\%). Umgekehrt kamen die US-amerikani-

8 Eine knappe Zusammenfassung der Argumente bzw. Gründe für Zusammenschlüsse und Größe in der Pharmaindustrie sowie Hinweise, warum manche Unternehmen nicht dem Trend folgen, finden sich bei D. Green (1998a und b). 
Abbildung 2 Pharmazeutische Exporte ${ }^{a}$ in Mio. US-\$ (logarithmische Skalierung)

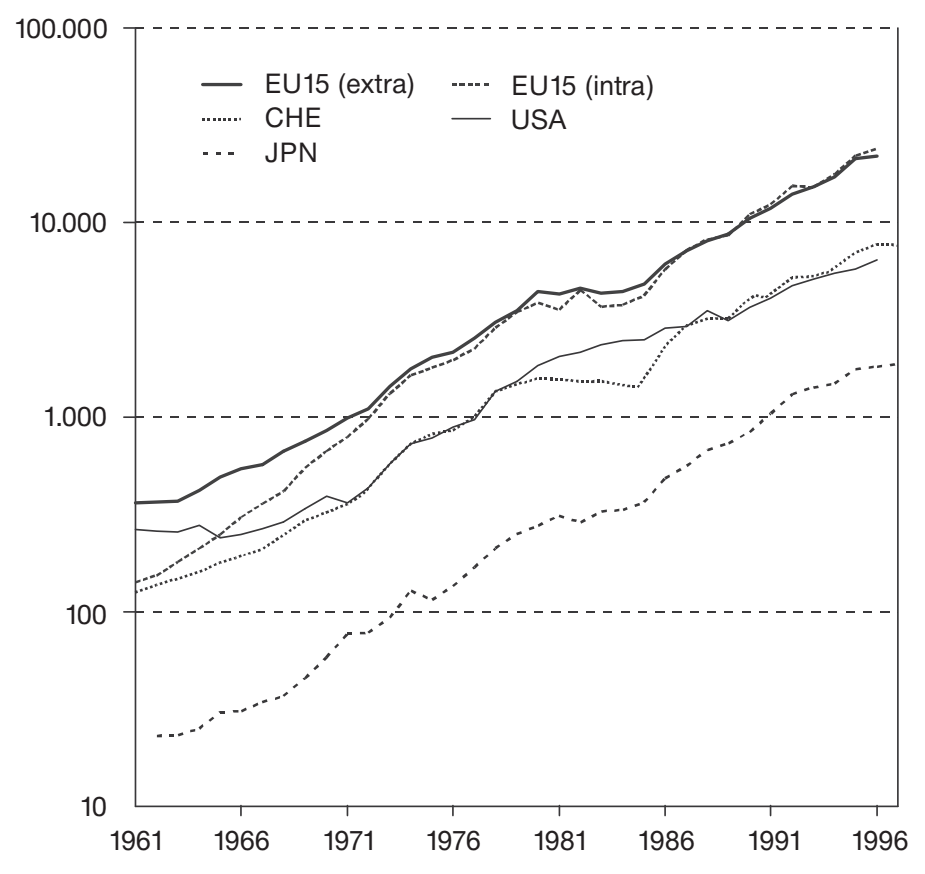

a ITCS Kategorien: 54 ohne 5419.

Quelle: OECD (Hg.): International Trade by Commodities Statistics, 1961-1990 \& 1998.

schen Unternehmen 1993 auf 6,4\% des japanischen Marktes, die europäischen auf 7,3\%. Während letztere relativ konstante Anteile hielten, gingen die der US-Unternehmen fast auf die Hälfte zurück. ${ }^{9}$

Seit Anfang der Sechzigerjahre sind die Ausfuhren in allen drei Regionen/Ländern im Großen und Ganzen parallel angestiegen und haben sich etwa alle fünf Jahre verdoppelt (Abbildung 2). Für den EU15-Raum gilt, dass seit Anfang der Siebzigerjahre die Ausfuhren innerhalb dieser Region genauso hoch ausfallen wie die außerhalb der EU-Grenzen und vom absoluten Niveau her weit über den Exporten der USA liegen. ${ }^{10}$ Ein differenzierteres Bild ergibt sich bei der Betrachtung der relativen Bedeutung des Exports für die jeweilige nationale bzw. regionale Pharmaindustrie (Abbildung 3). Hier zeigt sich, dass die Pharmaindustrie innerhalb des europäischen

9 Da bei diesen Veränderungen unterschiedliche Faktoren eine Rolle spielen können, etwa die Auswirkungen von Wechselkursschwankungen oder die so genannter ,Handelskriege‘, sollten die Zahlen nur als grobe Anhaltspunkte gelesen werden.

10 Die wichtigsten Pharmaländer Europas (D, GB, F; dazu CH) liegen im Export einzeln auf etwa dem gleichen Niveau wie die USA. 
Abbildung 3 Anteil der Exporte ${ }^{a}$ an der pharmazeutischen Produktion ${ }^{b}$

$\%$

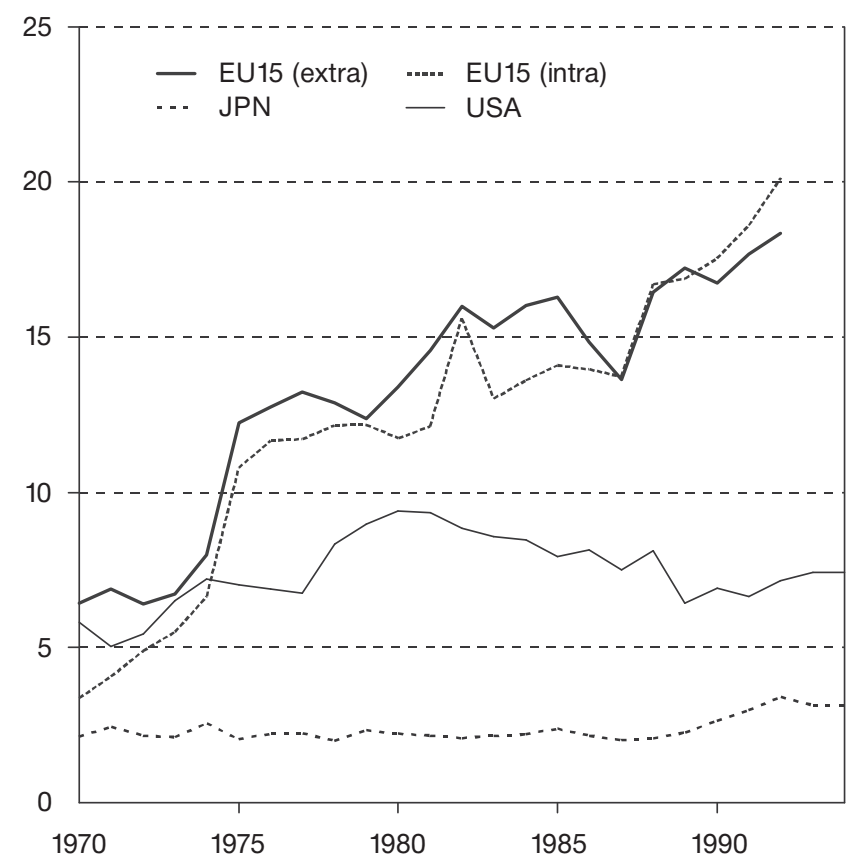

Berechnungsbasis = US-\$

EU15: ohne Irland; 1970-1975 ohne Deutschland; ab 1988 ohne Italien; Daten für 1992-1994 unvollständig.

a ITCS Kategorien: 54 ohne 5419. Quelle: OECD (Hg.): International Trade by Commodities Statistics, 1961-1990 \& 1998.

b Source OECD Health Data 1998.

Raumes (EU) seit den Siebzigerjahren im Gegensatz etwa zu den USA deutlich exportabhängiger geworden ist.

Die Exportabhängigkeit regionaler und nationaler Pharmaindustrien, die gegenseitige Durchdringung der Pharmamärkte wie auch die internationale Standortbreite großer pharmazeutischer Unternehmen lassen auf ein erhebliches Interesse europäischer Unternehmen an einer Vereinheitlichung regulativer Bedingungen des Marktzugangs innerhalb und außerhalb wichtiger Exportregionen Europas schließen, welche potenziell die regulativen Kosten vermindern. Ein etwas geringeres ,Harmonisierungsinteresse' liesse sich bei den USA und ein weiter vermindertes bei Japan vermuten; bei Japan ist allerdings die grundsätzliche Exportorientierung der japanischen Volkswirtschaft und eine langfristig an Exportvereinfachung im Kosmetikund Pharmabereich angelegte Strategie zu berücksichtigen. 


\section{Marktkonstituierung durch Intervention: \\ Die Regulierung der Arzneimittelzulassung}

Im Folgenden werden am Beispiel der Arzneimittelzulassung sehr vereinfachend Kernpunkte der Regulierungsentwicklung in den drei europäischen Ländern unter Einbeziehung der US-amerikanischen Situation dargestellt. Dabei sollen weniger die nationalen Differenzen im Vordergrund stehen, sondern internationale Anpassungsprozesse bis hin zu transkontinentalen Harmonisierungsbemühungen und nicht zuletzt die Herausbildung einer supranationalen Regulierung auf europäischer Ebene nachgezeichnet werden. Abschließend werden die Rückwirkungen von Internationalisierungsprozessen auf nationale Regulierungs- und Implementationsstrukturen aufgezeigt.

\subsection{Regulierung zwischen nationaler Differenz und internationaler Annäherung}

Der Zugang zu Heilmitteln in einem ganz allgemeinen Sinne war bereits vor Jahrtausenden sozial vermittelt. Das galt für die innerhalb von Familien oder Clans tradierten ,Hausmittel` wie auch für ,Arzneien` und Anwendungen, die spezialisierte Medizinmänner - oder wie immer die meist kultisch legitimierten Sachwalter genannt wurden - für größere Gemeinschaften verabreichten und die ihr Wissen häufig an Nachfolger innerhalb des gleichen Familienverbandes weitergaben. Bereits hier spielte die Annahme einer spezifischen, allerdings personengebundenen Expertise eine wichtige Rolle. Später, mit zunehmendem und dann auch systematisiertem Erfahrungswissen über die Wirkungsweise gesammelter und/oder zubereiteter Substanzen, bildeten sich spezialisierte Professionen (Ärzte, Apotheker) heraus, die zunächst mit tradierten Vorrechten, später kodifizierten Rechten der Herstellung, Lagerung, dem Vertrieb und der Verordnung versehen und zunehmend detaillierten Regelungen unterworfen wurden. Die staatlichen Vorgaben blieben Jahrhunderte lang inhaltlich außerordentlich rudimentär und die Kontrollen waren überwiegend als professionelle Selbstregulierung auf der Basis prozeduraler Vorgaben verankert (vgl. hierzu R. Schmitz 1998; G. Stille 1994). Später wurde diese durch eine zunehmend ausgefeilte Produkt- und Prozessregulierung ergänzt, die sich auf die Herstellung, Lagerung und den Vertrieb von Arzneimitteln konzentrierte. Bis zu Beginn des 20. Jahrhunderts verblieb der Marktzugang jedoch mehr oder weniger in industrieller Selbstkontrolle.

Spätestens seit den Sechzigerjahren zeichnete sich eine schrittweise Institutionalisierung und zunehmende Verschärfung von Marktzugangskontrollen ab. Als Folge der industriellen Herstellung und zunehmenden Massenverbreitung so genannter Fertigarzneimittel stiegen die Risiken von Arzneimittelunfällen über lokale Grenzen und zahlenmäßig kleine Betroffenenkreise hinaus erheblich an. Besonders sichtbar wurde dies an Katastrophen wie dem Thalidomid(Contergan)-Skandal der frühen Sechzigerjahre, der auf Medikamenteneinnahme zurückging und einen politischen Handlungsdruck erzeugte, dem sich die Verantwortlichen letztlich kaum entziehen 
konnten. ${ }^{11}$ Gleichwohl zeigt ein internationaler Überblick jedoch, dass einzelne Länder unterschiedlich schnell und auch unterschiedlich durchgreifend mit neuer Regelbildung auf derartig öffentlichkeitswirksame Katastrophen reagierten (vgl. Abbildung 4).

In Frankreich fiel es einem interventionistisch orientierten Staat offenbar recht leicht, die während des Zweiten Weltkriegs aus kriegswirtschaftlichen Gründen 1941 eingeführte formelle Zulassungsregelung (,visa“) beizubehalten, was auch an der politisch wenig durchsetzungsfähigen, weitgehend mittelständisch organisierten Arzneimittelindustrie lag. Jedoch erst nach der so genannten Stalinon-Affäre der Fünfzigerjahre (Grund: ein toxisch verseuchtes Vitamin-Präparat) wurden dieser Zulassungsregelung auch die notwendigen Verfahrensrichtlinien zur Seite gestellt (N. Reich 1988: 25-28).

In den USA schlug öffentlich gewordene Besorgnis besonders schnell auf den politischen Prozess durch - hier führte die so genannte Sulfanilamid-Affäre von 1937 (Grund: ein antibiotisches, auf Licht toxisch reagierendes Baby-Puder) zur Verankerung von Qualitäts- und Toxizitätskontrollen als Zulassungsbedingungen von Medikamenten.

Verglichen mit Frankreich und den USA waren die Ansätze zu effektiven Regelungen der Arzneimittelzulassung in Deutschland und Großbritannien vor der Thalidomid-Katastrophe wesentlich bescheidener. Dies lag u.a. an der in beiden Ländern sehr ausgeprägten wirtschaftspolitischen Bedeutung der Pharmaindustrie sowie einem kooperativen Verhältnis zwischen Industrie und Regierung, welches von der Öffentlichkeit weitgehend abgeschirmt war. Fragen der Produktqualität und -sicherheit blieben folglich lange Zeit industrieller Eigenverantwortung überlassen. So kam es in Großbritannien selbst nach der Thalidomid-Katastrophe zunächst nur zu einer Empfehlungsregelung, bevor schließlich in enger Abstimmung mit Industrieinteressen 1968 kodifiziertes Recht entstand (L. J. Smith 1991: 20-30; J. Abraham 1995: 56-64). In Deutschland scheiterten entsprechende Forderungen seit Ende des 19. Jahrhunderts regelmäßig an industriellen und politischen Widerständen, sodass es in den Zwanzigerjahren nur zu einer nichtstaatlichen Kooperation zwischen Ärzten und Krankenkassen kam (A. Murswieck 1983: 412-435). Auch das Arzneimittelgesetz (AMG) von 1961 - noch vor der Contergan-Affäre erlassen - und dessen Novellierung 1964 (Contergan-Novelle) führten letztlich nur zu einer Registrierung, nicht aber substanziellen staatlichen Evaluierung von Zulassungsunterlagen und formellen Zulassung von Medikamenten vor der Vermarktung. Es bedurfte nicht nur der Contergan-Katastrophe, sondern auch der Öffentlichkeitswirkung eines neuerlichen Arzneimittelunfalls (Menocil), um die Widerstände auch der Industrie gegen die Vorschrift substanzieller Arzneimittelprüfungen sowie deren staatliche Evaluierung zu brechen. Einen gesetzgeberischen Schub brachte zusätzlich die Regierungsumbildung von 1969 (von der CDU/SPD- zur SPD/FDP-Koalition).

11 Die historischen Abschnitte in J. Abraham (1995) geben einen Eindruck von der Viefalt der Faktoren, die zur Einführung oder Verschärfung von Zulassungs- und Überwachungsregelungen beitrugen. 


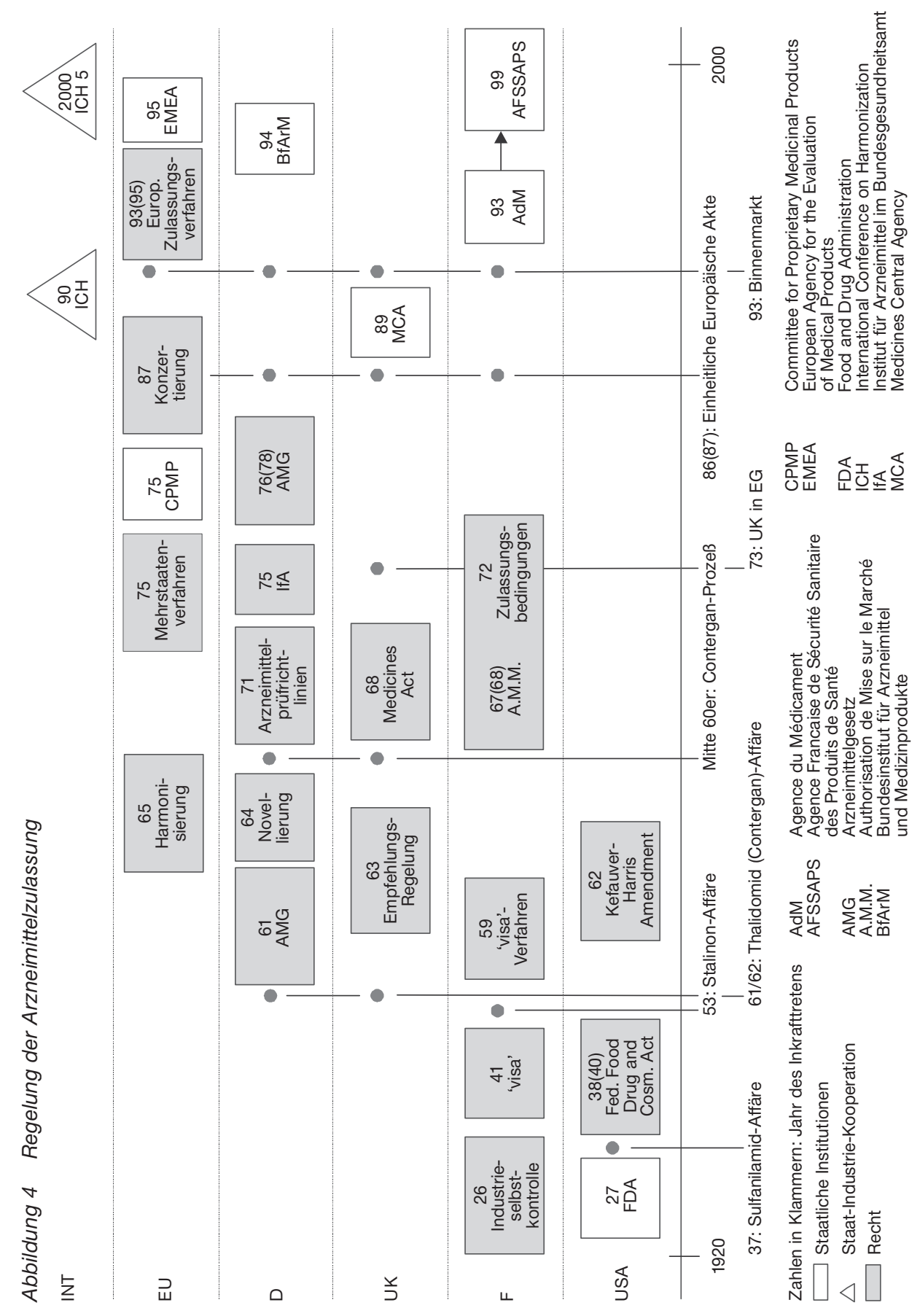


Resultat waren die Prüfrichtlinien von 1971 und schließlich das AMG von 1976, das 1978 in Kraft trat und die Bundesrepublik im Hinblick auf die materielle Zulassungsprüfung als Letztes der hier untersuchten Länder auf die Höhe der damaligen Zeit brachte.

Trotz unterschiedlicher historischer Pfade entwickelten die hier betrachteten Staaten im Laufe der Sechziger- und Siebzigerjahre ganz ähnliche rechtliche Regelungen zur Kontrolle der Arzneimittelzulassung. ${ }^{12}$ Wachsender Problemdruck durch die Gefährdung der öffentlichen Gesundheit, aussenwirtschaftlich schädliche Auswirkungen komparativer regulativer Rückständigkeit sowie die Vorbildfunktion einzelner Vorreiterstaaten erwiesen sich als zentrale Antriebsfaktoren dieses Konvergenzprozesses. Die Binnenmarktzielsetzung der EG entwickelte seit Anfang der Sechzigerjahre eine zusätzliche Dynamik, die schließlich in supranationaler Regulierung mündete. ${ }^{13}$

\subsection{Europa - Der Weg zur national flankierten Supranationalität}

Im Hinblick auf die Arzneimittelzulassung stellt die Europäische Gemeinschaft eine besonders interessante Regulierungsarena dar (D. Hart 1988). Obgleich der Gesundheitsbereich in den Römischen Verträgen - mit Modifikationen seither - explizit der Verantwortungshoheit der Nationalstaaten überlassen wurde, gab es gerade im Hinblick auf die Produktzulassung in der Arzneimittelregulierung schon frühzeitig Harmonisierungsversuche (D. Hart/N. Reich 1990), die in entsprechende Direktiven und Richtlinien einmündeten. Zunächst sollten hier im Sinne einer negativen Integration $^{14}$ all solche Handelshemmnisse ausgeräumt werden, die nicht den Schutz der öffentlichen Gesundheit als nationalen Rechtfertigungsgrund für sich in Anspruch nehmen konnten. Die Europäische Kommission nutzte das bereits erwähnte Thalidomid-Desaster der frühen Sechzigerjahre, um die auch in der US-Gesetzgebung enthaltenen Kriterien der Qualität, Sicherheit und - nun zusätzlich - Wirksamkeit als Voraussetzungen für eine aktive Zulassungspolitik der Mitgliedstaaten zu verankern. Die nationalen Regelungen der EG-Mitgliedstaaten wurden diesen seit 1965 verabschiedeten Direktiven und Richtlinien sukzessive angepasst, welche selber das Ergebnis eines intensiven Informationsprozesses und Dialogs zwischen den im Arzneimittelbereich wichtigeren EG-Staaten waren, der den Blick über den Atlantik einschloss. Trotz eines am Ende hohen formalen Harmonisierungsgrades auf der Policy-Ebene innerhalb der EG ${ }^{15}$ war diese Strategie nicht erfolgreich. Nach

12 Ähnlichkeit bedeutet hier nicht die Aufhebung sämtlicher Länderdifferenzen, vielmehr bestanden und bestehen solche in spezifischen Regelungsinhalten, Entscheidungsstrukturen und Implementationsstilen fort; vgl. R. Mayntz / J. Feick (1982); A. Murswieck (1983); D. Hart et al. (1988); N. Reich (1988); L. Hancher (1990); U. Baumheier (1993); J. Abraham (1995).

13 Für die gegenseitige Beeinflussung nationaler Regelungen im Arzneimittelbereich wie auch die Einflüsse internationaler Organisationen siehe für Deutschland H. Hasskarl (1978: 74) und A. Murswieck (1983: 432-435); genereller G. Majone (1990) sowie L. Hancher/ M. Moran (1989).

14 Siehe hierzu F. Scharpf (1997).

15 Auch die britische Gesetzgebung - Großbritannien war noch nicht EG-Mitglied - stand im Sog die- 
wie vor kam es nicht zur gegenseitigen Anerkennung nationaler Zulassungsentscheidungen, zudem konterkarierten nationale Implementationsdifferenzen die angestrebte Harmonisierung.

In der nächsten Phase führte man europäische Verfahren der Arzneimittelzulassung ein (Mehrstaatenverfahren 1975; neues Mehrstaatenverfahren oder Konzertierungsverfahren 1987) und richtete einen Ausschuss für Arzneimittelspezialitäten (CPMP) auf europäischer Ebene ein. Dies waren Maßnahmen, die in Fällen von Differenzen zwischen nationalen Zulassungsbehörden den Prozess gegenseitiger Anerkennung bzw. Anpassung von Zulassungsentscheidungen durch gemeinsame Beratungen erleichtern sollten, jedoch klar unterhalb der Schwelle supranationaler Entscheidung verblieben. Auch dieser Weg erwies sich als wenig erfolgreich. Der von Repräsentanten aller nationalen Behörden besetzte europäische Ausschuss konnte nur Empfehlungen an die nationalen Behörden aussprechen, die für ihr Territorium weiterhin die Letztentscheidung behielten. Sie nutzten ihre Vetorechte dazu, von den Antragstellern Nachuntersuchungen einzufordern und machten das supranationale Zulassungsverfahren für diese unattraktiv. Differenzen in der Sicherheitsphilosophie, Misstrauen gegenüber den Behörden anderer Länder, versteckter Protektionismus oder auch das organisatorische Eigeninteresse nationaler Behörden mögen die Motive dieser nationalen Widerstände gewesen sein. Im Grunde zeigten diese Hindernisse, dass nur ein Verfahren die gewünschte Marktintegration bringen konnte, welches den nationalen Behörden ihre Veto-Macht nehmen würde. ${ }^{16}$ Dem entspricht auch Majones Hinweis, dass bei Abwesenheit gegenseitigen Vertrauens in die Regulations- und Implementationspraxis des jeweiligen Landes „centralisation of regulatory authority“ die einzige praktikable Möglichkeit sei, „of correcting transboundary externalities, or preventing the local regulation of a local market failure from becoming a trade barrier“ (G. Majone 1998: 32). Das sukzessive Versagen vorhergehender Lösungen war es letztlich, was zur Einführung originär europäischer Zulassungsverfahren 1995 führte ${ }^{17}$ - einem zentralisierten für Medikamente der Biotechnologie (obligatorisch) sowie andere innovative Produkte (fakultativ) und einem Mehrstaatenverfahren mit gegenseitiger Anerkennung für alle anderen Präparate bei letztendlicher Entscheidungshoheit der europäischen Ebene.

Die Zentralisierung regulativer Entscheidungsgewalt auf europäischer Ebene als stärkste Form der Koordination von Gemeinschaftspolitik im Sinne eines „European maximalism“ erscheint hier aus funktionalistischen Gründen durchaus wünschenswert ${ }^{18}$. Diese liegen in Handelsbarrieren durch lokale Regulierung und damit der Verhinderung des Zugangs zu Medikamenten (Patientenperspektive) und zu Märk-

ser internationalen Anpassung.

16 Diese Defizite wurden bereits Ende der Achtzigerjahre intensiv diskutiert, ebenfalls die Lösungsmöglichkeit durch eine supranationale Behörde, die schließlich von der EG-Kommission und nach Zögern auch von Teilen der Industrie favorisiert wurde. Vgl. hierzu D. Hart/N. Reich (1990: 3740); ebenfalls E. Kaufer (1990).

17 Für die rechtlichen Details wie auch die vorausgegangenen Regelungen auf europäischer Ebene siehe zusammenfassend B. Collatz (1996) und E. Vos (1997).

18 Siehe hierzu F. Scharpf (1994: 239, 227). 
ten (Herstellerperspektive) wie auch in der Erhöhung von Transaktionskosten eines solchen Marktzugangs. Damit ist jedoch noch nicht erklärt, warum sich die einzelnen Staaten Anfang der Neunziger Jahre auf eine solche Lösung verständigt haben. Zumindest ein Grund kann in der spezifischen institutionellen Entscheidungsprozedur gesehen werden. Bei aller Zentralisierung sind die nationalen Regulierungsakteure in einer Weise in das Entscheidungssystem einbezogen, welche Konfliktkanalisation und Konsensbildung erleichtert.

Institutionell erscheint das CPMP von besonderer Bedeutung. Dieses Gremium ist die zentrale Evaluierungsinstanz in einem von der Europäischen Agentur in London koordinierten Zulassungsprozess. Im CPMP sitzen ad personam Vertreter aller nationalen Zulassungsbehörden, die sich monatlich für eine Woche zu Beratungen in London treffen. Trotz ihrer Weisungsungebundenheit sind diese Mitglieder an ihren jeweiligen nationalen, professionellen und institutionellen Hintergrund rückgebunden. Gleichzeitig erlaubt der wissenschaftlich und technisch geprägte Gegenstandsbereich der Arzneimittelzulassung in diesem nicht öffentlich tagenden Expertenkreis eine auch bei Meinungsunterschieden sachbezogene Diskussion. Hier findet also auf der wissenschaftlichen Evaluationsebene eine Integration nationaler Inputs in einen international besetzten und supranationale Entscheidungen vorbereitenden Expertenkreis statt. Auf der Basis der CPMP-Empfehlung wird die politisch-administrative Zulassungsentscheidung von der Kommission in Brüssel gefällt, die selbst von einem Ständigen Arzneimittelkomitee beraten wird, welches aus Vertretern ebenfalls der politisch-administrativen Ebene der Heimatbehörden bzw. -ministerien besteht. Diese sind explizit als Ländervertreter in dem Gremium. ${ }^{19}$

Kurzum: Das europäische Verfahren durchläuft verschiedene Filter, an denen alle nationalen Behörden beteiligt sind und innerhalb derer sie eventuelle Einwände und Bedenken zur Diskussion stellen können. Damit sind nationale Inputs sichergestellt - und zwar auf wissenschaftlich-evaluativer wie auf administrativ-regulativer Ebene. Von besonderer Bedeutung ist natürlich, dass diese Einflussmöglichkeiten nicht mit nationalen Vetorechten verbunden sind und hierdurch ein Zwang zur Konsensbildung bzw. zur Mehrheitsfindung besteht.

Institutionell wichtig ist außerdem, dass es für die Arzneimittelhersteller weiterhin die Möglichkeit gibt, Zulassungsanträge für nur ein Land zu stellen. Damit sind die nationalen Unternehmen geschützt, die nur für einen nationalen Markt produzieren und mit ihren Produkten möglicherweise Zulassungsschwierigkeiten in anderen Ländern und damit im dezentralisierten bzw. im zentralisierten europäischen Verfahren befürchten müssten. Auch dieses nationale Verfahrensresiduum dürfte den Mitgliedsländern die Akzeptanz einer supranationalen, europäischen Lösung erleichtert haben. Die Kombination dieser unterschiedlichen Verfahrenswege und die intensive organisatorische Partizipation der nationalen Behörden an den europäischen Zulassungsverfahren lässt zum einen den nicht auf internationale Märkte

19 Die Mitglieder dieses Ständigen Kommitees kommen seltener zusammen und regeln die meisten Abstimmungen auf schriftlichem Wege. 
konzentrierten kleineren und mittleren Unternehmen genügend Spielraum, befriedigt gleichzeitig die Effizienzwünsche der international operierenden Pharmaindustrie und kommt außerdem den organisatorischen Eigeninteressen der nationalen Behörden - in der Vergangenheit überwiegend Träger des Widerstands gegen die Supranationalisierung - entgegen.

Prinzipiell bleibt festzuhalten, dass die Arzneimittelzulassung zu den wenigen regulativen Bereichen der Europäischen Gemeinschaft gehört, in denen positive Integration möglich und zumindest prozedural auch weitgehend erfolgreich war. ${ }^{20}$ Mit Blick auf die regulativen Kosten für die Industrie kann von einem Nettogewinn und für die europäische Integration von einem symbolischen Gewinn gesprochen werden. Im Laufe der Jahrzehnte hat sich eine Politik herausgeschält, die nicht in dem von Streeck für zwei Teilbereiche der Europäischen Sozialpolitik (gleiche Rechte für Frauen in der Arbeitswelt; arbeitsplatzbezogener Gesundheits- und Sicherheitsschutz) diagnostizierten „encapsulated federalism“ (W. Streeck 1995: 45) stecken geblieben ist. Letzterer steht für die Entwicklung supranationaler Ansätze, die in der Folgezeit von nationalen und intergouvernementalen Handlungsmöglichkeiten so ummantelt werden, dass praktisch nichts mehr von der supranationalen Dynamik übrig bleibt, denn „,...) the influence of supranational social policy (...) is confined by its dependence on the voluntarism of both sovereignty-conscious member states and interest-conscious private market participants“ (W. Streeck 1995: 57f.).

Im Bereich der Zulassungsregulierung ist eine kaum noch rückholbare Supranationalisierung zu beobachten, in der durch institutionelle und prozedurale föderalistische Gegengewichte die Partizipation nationaler Interessen gesichert wird, ohne die gewollte supranationale Dominanz in Frage zu stellen. Man könnte hier von einem „procedurally balanced federalism“ sprechen. Im vorliegenden Beispiel haben intergouvernementale und daran sich anschließende supranationale Entscheidungen zum Aufbau und zur Festigung regulativer Strukturen geführt, die den bisherigen nationalen Regelungen zumindest formal nicht nachstehen und sogar dazu tendieren, die national höchsten Vorgaben supranational abzusichern. Relativierend muss jedoch auf eine zunehmende Klientenorientierung der Europäischen Behörde und des europäischen Zulassungsprozesses hingewiesen werden, welche auch nationale Regulierungsbehörden unter Effizienzdruck setzt. Ob eine industriefreundliche Zulassungspolitik auch zu einer Aufweichung von Zulassungskriterien im Implementationsprozess führt, kann derzeit noch nicht abschließend beurteilt werden. ${ }^{21}$

20 Im Jahr 2001 ist eine Evaluierung durch die EU-Kommission vorgesehen.

21 Vgl. hierzu eine Umfrage unter Mitgliedern verschiedener nationaler Zulassungsbehörden mit unterschiedlichen Einschätzungen in J. Abraham/G. Lewis (1999). 


\subsection{Internationale Harmonisierung jenseits von Europa}

Regulative Harmonisierungsbemühungen gehen mittlerweile über den EU-Raum hinaus. Diese zielen darauf ab, die Zulassungsbedingungen zwischen den Ländern der großen regionalen Märkte zu harmonisieren, welche zusammen etwa 90 Prozent des Weltpharmamarktes repräsentieren und wechselseitige Exportinteressen haben (vgl. Abschnitt 3). Wie innerhalb der Europäischen Union mit ihrem hohen internen Warenaustausch im Pharmabereich, so ist auch auf internationaler Ebene bei den exportierenden Unternehmen ein Interesse vorhanden, die regulativen Kosten zu reduzieren. Nationale Zulassungsverfahren mit je spezifischen Anforderungen an die vorzulegenden Zulassungsunterlagen und darin enthaltenen Analyse-, Testund Prüfergebnisse erhöhen die Kosten für international agierende Unternehmen nicht nur im Zulassungsprozess, sondern bereits bei der Beschaffung der verlangten Informationen im Forschungs- und Entwicklungsprozess.

Im Mittelpunkt dieser Bemühungen um eine Harmonisierung solcher Anforderungen steht die seit 1990 eingerichtete International Conference on Harmonization (ICH), in der Repräsentanten der Regulierungsbehörden der EU, der USA und Japans sowie der entsprechenden nationalen bzw. regionalen Industrieverbände in einem Steuerungsausschuss (Steering Committee) zusammenarbeiten, koordinierend unterstützt vom Internationalen Verband der Pharmaverbände (IFPMA). Als Beobachter nahmen von Anfang an die entsprechenden Organisationen Kanadas und der EFTA-Länder teil. Die grundlegende Harmonisierungsarbeit wird von Experten aus der Industrie, den regulierenden Behörden und der Wissenschaft in einer Vielzahl von Arbeitsgruppen geleistet. Deren Arbeitsergebnisse durchlaufen einen mehrstufigen Abstimmungsprozess, ausgehend vom Steering Committee, über die Rückbindung an jeweils nationale, im Falle der EU europäische Gremien; schließlich werden alle Kommentare zu Richtlinien verarbeitet, die von den drei regulierenden Behörden der EU, der USA und Japans gemeinsam zu verabschieden sind. Zwar haben diese Beschlüsse nur empfehlenden Charakter für die beteiligten Zulassungsbehörden; aus der Beteiligung an der Harmonisierungsarbeit und der Zustimmung zum Diskussionsergebnis wird jedoch eine ,moralische‘ Implementationsverbindlichkeit abgeleitet.

Das Ziel war zunächst, durch die Standardisierung von Test- und Prüfverfahren die gegenseitige Anerkennung von Analyseergebnissen und Zulassungsunterlagen zu erreichen. In einem zweiten Schritt sollen die Inhalte von ,Zulassungsdossiers definiert werden, sodass es im Idealfall für jedes Produkt nur ein Antragsdossier geben müsste, welches alle Unterzeichnerbehörden anerkennen. Es geht also um eine Form gegenseitiger Anerkennung, die aber voraussetzt, dass vorausgegangene Harmonisierung Zweifel darüber ausräumt, was inhaltlich Gegenstand der Anerkennung ist. Bislang schwanken die Einschätzungen über den bisher erreichten Grad an Harmonisierung. Für die Attraktivität dieser Harmonisierungsarbeiten spricht die im Laufe der Jahre ständige Erweiterung des Beobachterkreises an Treffen der Arbeitsgruppen. Behindert werden die Abstimmungsversuche jedoch immer wieder durch die Neigung insbesondere der amerikanischen Regulierungsbehörde FDA, ihre Vorstellungen im Falle von Meinungsunterschieden zwischen den ver- 
schiedenen Experten mit Nachdruck durchsetzen zu wollen (J. Wechsler 1996). ${ }^{22}$ Ansonsten wird jedoch betont, dass die gemeinsam verabschiedeten Richtlinien, welche alle technisch-wissenschaftlich sehr detailliert sind, in der Regel Eingang in die Regulierungspraxis fänden.

Bei diesen Harmoniserungsansätzen bleibt unklar, unter welchen Bedingungen eine gegenseitige Anerkennung zwischen nationalen/regionalen Behörden ohne supranationale Durchsetzungsmittel gelingen kann - ein Problem, welches sich auch in der Anfangsphase der europäischen Harmonisierung von Zulassungsregeln zeigte. Die optimistischere Einschätzung entspringt einer eher funktionalistischen Logik, die davon ausgeht, dass dieser Prozess der internationalen Harmonisierung nicht aufzuhalten ist und sich mit der Internationalisierung von wirtschaftlichen Aktivitäten, insbesondere des Handelsaustauschs, quasi unabhängig vom konkreten institutionellen Unterbau ausbreiten wird. Der wissenschaftliche und technische Charakter dieser Harmonisierungsaufgabe würde zusätzlich für dieses Szenario sprechen. Umgekehrt verweisen die europäischen Erfahrungen darauf, dass erfolgreiche Harmonisierung der Arzneimittelzulassung eine so voraussetzungsvolle Aufgabe ist, dass deren Verfolgung mit einer gewissen Konsequenz auf supranationale Verfahren hinausläuft, wenn sie die in sie gesetzten Erwartungen erfüllen soll.

\subsection{Rückwirkungen der Internationalisierung auf die nationale Ebene: Die Verwaltung auf dem Weg zum Dienstleister}

Die zunehmende Internationalisierung des Pharmamarktes und der durch die regulative Supranationalisierung ausgelöste regulative Wettbewerb zwischen den beteiligten nationalen Behörden setzen nationale Verwaltungsstrukturen unter Anpassungsdruck. Regulierungsinstitutionen werden dazu angehalten, regulative Kosten für die antragstellende Industrie nicht unnötig zu erhöhen, um die Wettbewerbsfähigkeit ihrer Klientel nicht zu behindern. Im Kern geht es dabei um eine stärkere Marktorientierung und auch interne „Vermarktlichung“ der mit der Arzneimittelzulassung befassten nationalen Verwaltungen. Drei „Trends“ können ausgemacht werden, die in allen Ländern, wenn auch in unterschiedlicher Stärke, zu beobachten sind: Erstens, die Verselbständigung der regulativen Behörden; zweitens, ein Einstellungswandel von der Kontroll- zur Klientenorientierung; drittens, eine auf Effizienz zielende Organisationsperspektive. Weniger eindeutig sind die Konvergenzsignale, was die Beziehungen zur Öffentlichkeit angeht. Da diese Entwicklungen nicht unabhängig voneinander verlaufen sind, ergibt es Sinn, sie länderbezogen zu behandeln.

Am weitesten gingen die Veränderungen in Großbritannien, wo 1989 die alte Zulassungsabteilung (Medicines Division) im Gesundheitsministerium aufgelöst und die „Medicines Control Agency“ (MCA) als vollständig aus Gebühreneinnahmen finanzierte und damit in Budgetierungs-, Rekrutierungs- und Besoldungsfragen

22 Besondere Verstimmung hat eine nachträgliche Rücknahme der Zustimmung zu einer bereits empfohlenen Richtlinie durch die US-Behörde verursacht. 
freiere Institution (so genannter „Trading Fund Status“) gegründet wurde. Sie steht damit in allen operationell relevanten Managemententscheidungen außerhalb der hierarchisch-ministeriellen Entscheidungsstruktur, obgleich die MCA formell weiterhin dem Minister untersteht. Sie wird von einem eigenen Board kontrolliert und alle fünf Jahre einer veröffentlichten Evaluation durch das Gesundheitsministerium unterzogen.

Dieser organisatorisch-institutionelle Wandel ging einher mit der Einführung privatwirtschaftlicher Managementtechniken in öffentliche Institutionen, welche die Regierung Thatcher mit dem Leitbild der „new public administration“ verband. Seither versteht sich die MCA als effizienzorientierte Behörde, die ihre finanzielle und regulative Performanz kontinuierlich kontrolliert, dokumentiert und seit ihrer Gründung die formalen Effizienzergebnisse wie Evaluationsdauer, Einhaltung vorgegebener Zeitspannen und Termine stetig verbessert hat. Die Behörde sieht sich heute nicht mehr als legalistisch verfahrende Bürokratie mit Kontrollauftrag, sondern als Dienstleister, der seinen Kunden - in Zulassungsfragen der Pharmaindustrie - „value for money“ zu liefern habe. Dazu gehört es, die Unternehmen schon im Vorfeld zu beraten und neuerdings - früher undenkbar - auch während des Evaluierungsprozesses für Rückfragen zur Verfügung zu stehen. Die Industrie zeigt sich mit der bisherigen Bilanz der Behörde außerordentlich zufrieden. Gegenüber der Öffentlichkeit gilt sie als im traditionellen Sinne weiterhin eher verschlossen, was bei der beschriebenen Effizienz- und Klientenorientierung nicht überrascht. So genannte Pharmakritiker werfen der Behörde deshalb auch zu große Industrienähe und insbesondere eine zu geringe Transparenz aus der Sicht der Öffentlichkeit vor (vgl. hierzu J. Abraham/G. Lewis 1999).

Die Ausgangssituation war in Frankreich ganz ähnlich: Hier bestand eine in das Gesundheitsministerium integrierte Zulassungsabteilung, die von der Pharmaindustrie wegen schleppender Antragsbearbeitung und deshalb langen Zulassungszeiten ständig kritisiert wurde. In Anlehnung an die MCA wurde 1993 die „Agence du Médicament“ (AdM) gegründet. Sie untersteht weiterhin rechtlich der Aufsicht des Gesundheitsministers und ist budgetär in dessen Haushalt verankert. Sie finanziert sich auch nur zum Teil aus Gebühreneinnahmen. Trotzdem erlaubt ihr der relativ selbständige Status eine, verglichen mit der früheren Situation, grössere Freiheit in der Nutzung vorhandener Ressourcen. Sie ist für ihre Beschaffungs-, Einstellungswie ihre regulativen Entscheidungen nicht mehr an die ministerielle Hierarchie gebunden und kann entsprechend flexibler wirtschaften und organisieren. Mit der behördlichen Verselbständigung gingen in der AdM auch Veränderungen im Interaktionsverhalten zwischen Behörde und Industrie einher. Große Anstrengungen wurden unternommen, von einer traditionell etatistischen Kontrollorientierung zu einer eher professionell-kooperativen zu gelangen, deren etatistische Züge nun in gewisser Weise paternalistische Merkmale annehmen. So ist die französische Agentur die Einzige der nationalen Behörden, ${ }^{23}$ die in London eigens ein Büro unterhält, um

23 Die Briten haben hier natürlich den Vorteil, dass die europäische Behörde in London angesiedelt ist. 
den französischen Unternehmen vor Ort Unterstützung innerhalb der europäischen Verfahren geben zu können. Die Aussenbeziehungen zu einer breiteren Öffentlichkeit nehmen bei der französischen Behörde einen vergleichsweise grösseren Platz ein. Das hat nicht nur damit zu tun, dass neben einem Industrieverbandsvertreter auch ein Verbrauchervertreter (beide allerdings nicht stimmberechtigt) an den Sitzungen des Evaluationskomitees teilnimmt. Auch ein Detail wie die Veröffentlichung der direkten oder indirekten Industrieverbindungen der evaluierenden Experten weist auf eine bewusste Politik der Transparenz hin.

In der Bundesrepublik erwies sich eine solche formale Ausgliederung aus dem zuständigen Ministerium als nicht notwendig, war die deutsche Zulassungsinstitution doch bereits als Bundesinstitut Teil des Bundesgesundheitsamtes und nie in das Bundesministerium integriert. Nach der Auflösung des Bundesgesundheitsamtes im Jahr 1994 wurde die Regulierungsbehörde als „Bundesinstitut für Arzneimittel und Medizinprodukte (BfArM)“ dem Bundesgesundheitsministerium direkt unterstellt. Wie die französische Behörde finanziert sie sich zu etwa der Hälfte des Budgets aus Gebühreneinnahmen von der Industrie. Trotz der relativen Selbständigkeit hat das BfArM jedoch nie die flexible Manövrierfähigkeit der britischen Agentur erreicht, weder in finanzieller, noch in organisatorischer Hinsicht - Personalressourcen eingeschlossen. Auch die französische Behörde kann vergleichsweise unabhängiger verwaltet werden als die deutsche. Letztere verlor auch nie das Image einer eher bürokratisch orientierten Institution. Von Partnerorganisationen in Europa wird inzwischen anerkannt, dass die deutsche Zulassungsbehörde in letzter Zeit besonders grosse Anstrengungen unternommen habe, um sich gerade in den europäischen Verfahren als zuverlässig arbeitender Partner zu etablieren. Dass zumindest hier Verbesserungen gelungen sind, zeigt die deutliche Verkürzung der Zulassungszeiten zwischen 1990 und 1995 (K. E. Thomas et al., 1998).

Was die Beziehungen zur pharmazeutischen Industrie angeht, bietet sich ein zwiespältiges Bild. Einerseits hat die pharmazeutische Industrie von Anfang an starken Einfluss auf die deutsche Gesetzgebung genommen, andererseits kritisierte sie die Implementation fortwährend als zu bürokratisch, langsam und intransparent. Eher pharmakritische Beobachter bemängeln eine zu grosse Industrienähe - auch durch die Beteiligung von Industriewissenschaftlern in den beratenden Kommissionen - und eine zu geringe Härte gegenüber Medikamenten mit eher zweifelhaftem Wirkungsnachweis. ${ }^{24}$ Insgesamt lassen sich auch bei der deutschen Regulierungsbehörde Veränderungen hin zu stärkerer Klientenorientierung feststellen - etwa in der Frage der Informationspolitik gegenüber der Industrie -, die allerdings schwächer ausgeprägt zu sein scheint als in den beiden anderen Ländern. Gleichwohl ist das Verhalten der Organisation zunehmend an den Kooperationsanforderungen der neuen europäischen Verfahren und dem Vergleich mit der Performanz anderer Partnerorganisationen orientiert.

24 Dies hat nicht zuletzt mit dem im deutschen Gesetz milder gefassten Wirkungsnachweis zu tun; hiermit kam die deutsche Regierung im Gesetzgebungsverfahren Wünschen von Industrie und Vertretern besonderer Therapierichtungen nach. 


\section{Zusammenfassende Interpretation}

Die Regulierung des Marktzugangs für Arzneimittel hat eine lange Tradition, deren inhaltlich-materielle Verschärfung und institutionelle Verfestigung im nationalen Bereich vor allem perzipierten Gefahren für die öffentliche Gesundheit geschuldet ist. Mit der Industrialisierung der Arzneimittelproduktion um die Jahrhundertwende sind diese Gefahren angestiegen und haben sich in wiederkehrenden Arzneimittelkatastrophen manifestiert.

Die Ausbreitung des internationalen Pharmahandels und die Internationalisierung von Pharmaunternehmen haben die Kommunikation auch zwischen nationalen Regulierungsinstanzen gefördert und international regulative Anpassungsprozesse zunächst im Hinblick auf materielle Regelungsinhalte angestossen (vgl. auch D. Vogel 1998). Der überwiegend technische und wissenschaftliche Charakter des Regelungsgegenstands hat diese Kommunikations- und Ausbreitungsprozesse erleichtert. Das Ergebnis sind regulative Konvergenzen, zunächst im Sinne gegenseitiger, ungesteuerter nationaler Anpassung bis hin zu international vereinbarten Standards oder auch einer zunächst intergouvernementalen, dann supranationalen Institutionalisierung von Kontrollen im Rahmen der Europäischen Union.

Gegenläufige Kräfte einer internationalen regulativen Konvergenz oder gar Supranationalisierung liegen im eher am heimischen Markt orientierten Teil der pharmazeutischen Industrie, aber auch in differierenden, nationalen regulativen Traditionen oder materiellen Regelungsphilosophien. Nicht zu unterschätzen sind auch aus organisatorischen Bestandsinteressen der nationalen Institutionen herrührende Widerstände.

Innerhalb der Europäischen Gemeinschaft wurden diese widerstrebenden Kräfte nicht nur durch die sich vervielfältigenden wirtschaftlichen Interdependenzen und den daraus entstehenden Druck der exportorientierten Wirtschaft allmählich zurückgedrängt, sondern auch oder institutionell sogar in erster Linie durch die politisch gewollte Schaffung eines Binnenmarktes. Dazu bedurfte es aber auch eines langwierigen Gewöhnungs- und Vertrauensbildungsprozesses durch zunehmende Kommunikation und Kooperation zwischen nationalen Behörden. Ob es jenseits der Europäischen Union zu Anpassungsprozessen, Konvergenzen und regulativen Integrationsprozessen kommen wird, die auch die nationale Umsetzung transnational vereinbarter Regelungsinhalte garantieren, bleibt derzeit ungewiss.

Das Implementationsverhalten der regulierenden Behörden hat sich, jedenfalls in wichtigen Ländern der Europäischen Union, in den letzten Jahren erheblich verändert. Angesichts von Industriekritik an ineffizienten und für sie dadurch kostspieligen Zulassungsverfahren, vor dem Hintergrund wirtschaftlicher Wachstumsprobleme und der Konzentration nationaler Industriepolitik auf innovative Industriebereiche werden regulierende Institutionen zunehmend daraufhin untersucht, ob sie die regulativen Kosten unnötig erhöhen und dadurch wirtschaftliches Wachstum bremsen. Zudem setzt ein immer schärferer regulativer Wettbewerb zwischen nationalen Behörden innerhalb der europäischen Zulassungsverfahren diese unter Effizienzdruck. Darin ist eine Ursache für den Wandel weg von bürokratischen Kontroll- und hin zu 
einer auch interne Effizienz betonenden Dienstleistungsorientierung gegenüber der zu kontrollierenden Industrie zu sehen.

Von den Marktzugangsbedingungen her gedacht, heißt dies, dass den beantragenden Unternehmen der Weg mit ihrem Produkt zum Markt prozedural erleichtert wird. Ob diese Zugangserleichterung rein prozedural bleibt und die materiellen $\mathrm{Zu}$ lassungshürden unberührt lässt oder vielmehr hierdurch Sicherheitsstandards aufgeweicht werden, wird derzeit breit diskutiert. Die einen erwarten von der regulativen Internationalisierung und dem regulativen Wettbewerb zwischen nationalen Behörden eine effektivere und effizientere Regulierung bei zumindest gleich bleibendem Schutzniveau (vgl. D. Vogel 1998: 20). Auf der anderen Seite gibt es Stimmen im Lager der so genannten Pharmakritiker, aber auch von Seiten mancher nationaler Zulassungsbehörden, die befürchten, dass ein zu klientenorientiertes Implementationsverhalten zur Vernachlässigung der Schutzfunktion gegenüber den Verbrauchern führt (J. Abraham/G. Lewis 1999).

\section{Literatur}

Abraham, John (1995): Science, Politics and the Pharmaceutical Industry. Controversy and Bias in Drug Regulation. London: UCL Press

Abraham, John/Lewis, Graham (1999): Harmonising and Competing for Medicines Regulation: How Healthy are the European Union's Systems of Drug Approval. In: Social Science \& Medicine 48. 1655-1667

Bator, Francis (1958): The Anatomy of Market Failure. In: The Quarterly Journal of Economics 72. 351-379

Baumheier, Ulrike (1994): Staat und Pharmaindustrie. Sicherheitskontrolle, Preisregulierung und Industrieförderung im internationalen Vergleich. Baden-Baden: Nomos

Collatz, Brigitte (1996): Die neuen europäischen Zulassungsverfahren für Arzneimittel: Insbesondere Verfahren und Rechtsschutz des Antragstellers und Zulassungsinhabers bei Zulassungsentscheidungen. Aulendorf: Editio Cantor Verlag

Evers, Paul (1998): Global Pharma Markets. London: Financial Times Professional Ltd.

Feick, Jürgen (1980): Zur Kritik regulativer Politik in den Vereinigten Staaten. In: Politische Vierteljahresschrift $21.43-61$

Green, Daniel (1998a): A Growing Dilemma. In: Financial Times, Financial Times Survey: Pharmaceuticals, March 16, 1998

Green, Daniel (1998b): Uniting Against a Sea of Troubles. In: Financial Times, Financial Times Survey: Pharmaceuticals, March 16, 1998

Hancher, Leigh (1990): Regulating for Competition: Government, Law, and the Pharmaceutical Industry in the United Kingdom and France. Oxford: Clarendon Press

Hancher, Leigh/Moran, Michael (1989): Organizing Regulatory Space. In: Hancher, Leigh/Moran, Michael (Hrsg.) (1989), Capitalism, Culture and Economic Regulation. Oxford: Clarendon Press: 271299

Hart, Dieter et al. (Hrsg.) (1988): Das Recht des Arzneimittelmarktes. Baden-Baden: Nomos

Hart, Dieter/Reich, Norbert (1990): Integration und Recht des Arzneimittelmarktes in der EG. BadenBaden: Nomos

Hasskarl, Horst (1978): Die internationale Interdependenz des neuen deutschen Arzneimittelrechts. In: Helmchen, Hanfried/ Müller-Oerlinghausen, Bruno (Hrsg.) (1978): Psychiatrische Therapie-Forschung. Berlin: Springer-Verlag: 61-77

Kaufer, Erich (1990): The Regulation of New Product Development in the Drug Industry. In: Giandomenico Majone (Hrsg.) (1990): Deregulation or Re-Regulation? Regulatory Reform in Europe and the United States. London/New York: Pinter Publishers/St. Martin’s Press: 153-175

König, Klaus/Dose, Nicolai (Hrsg.) (1993): Instrumente und Formen staatlichen Handelns. Köln: Carl Heymanns Verlag 
Majone, Giandomenico (1990): Cross-National Sources of Regulatory Policy-making in Europe and the United States. EUI Working Paper No. 90/6. San Domenico: European University Institute

Mayntz, Renate/Feick, Jürgen et al. (Hrsg.) (1982): Regulative Politik und politisch administrative Kultur. Ein Vergleich von fünf Ländern und vier Interventionsprogrammen. Institut für angewandte Sozialforschung. Köln

Müller, Jürgen/Vogelsang, Ingo (1979): Staatliche Regulierung. Regulated Industries in den USA und Gemeinwohlbindung in wettbewerblichen Ausnahmebereichen in der Bundesrepublik Deutschland. Baden-Baden: Nomos

Murswieck, Axel (1983): Die staatliche Kontrolle der Arzneimittelsicherheit in der Bundesrepublik und in den USA. Opladen: Westdeutscher Verlag

Nordmann, Ronald M. (1997): A Quarter Century of Monumental Change. In: Scrip Magazine, April 1997. 28-29

Reich, Norbert (1988): Arzneimittelregelung in Frankreich. Baden-Baden: Nomos

Roth, Martin S. (1997): New Strategies for a Global Industry: The Case of the Pharmaceutical Industry. In: Sabine Urban (Hrsg.) (1997): Europe in the Global Competition. Wiesbaden: Gabler: 193-214

Scharpf, Fritz W. (1994): Community and Autonomy: Multi-level Policy-making in the European Union. In: Journal of European Public Policy 1. 219-242

Scharpf, Fritz W. (1997): Balancing Positive and Negative Integration: The Regulatory Options for Europe. Policy papers. RSC No. 97/4. San Domenico: European University Institute

Silverman, Milton/Lee, Philip R. (1974): Pills, Profits and Politics. Berkeley: University of California Press

Schmitz, Rudolf (1998): Geschichte der Pharmazie. Eschborn: Govi-Verlag

Smith, Lesley Jane (1991): Legal Regulation of the British Pharmaceutical Market. Domestic Law, Internal Market and Intellectual Property Rights. Baden-Baden: Nomos

Stille, Günther (1994): Krankheit und Arznei. Berlin: Springer

Streeck, Wolfgang (1995): Neo-Voluntarism: A New European Social Policy Regime? In: European Law Journal 1. 31-59

Thomas, Kate E. et al. (1998): A Study of Trends in Pharmaceutical Regulatory Approval Times for Nine Major Markets in the 1990s. In: Drug Information Journal 32. 787-801

Vogel, David (1998): The Globalization of Pharmaceutical Regulation. In: Governance 11. 1-22

Vos, Ellen (1999): Institutional Frameworks of Community Health and Safety Legislation. Committees, Agencies and Private Bodies. Oxford: Hart Publishing.

Wechsler, Jill (1996): Washington vs. the World? In: Pharmaceutical Executive. February, 16-20 